\title{
Endoscopic treatment of Zenker's diverticulum by complete septotomy: initial experience in 19 patients $\square$
}

다 (i)

\author{
Authors \\ Dohan $^{2,3}$, Romain Coriat ${ }^{1,2}$, Stanislas Chaussade ${ }^{1,2}$ \\ Institutions \\ 1 Department of Gastroenterology, Cochin Hospital, \\ Assistance Publique-Hôpitaux de Paris, Paris, France \\ 2 University of Paris, France \\ 3 Department of Radiology, Cochin Hospital, Assistance \\ Publique-Hôpitaux de Paris, France
}

Charlotte Juin ${ }^{1}$, Maximilien Barret ${ }^{1,2}$, Arthur Belle ${ }^{1}$, Einas Abouali ${ }^{1,2}$, Sarah Leblanc ${ }^{1}$, Ammar Oudjit ${ }^{1,2}$, Anthony

submitted 7.1.2020

accepted after revision $\quad 25.3 .2020$

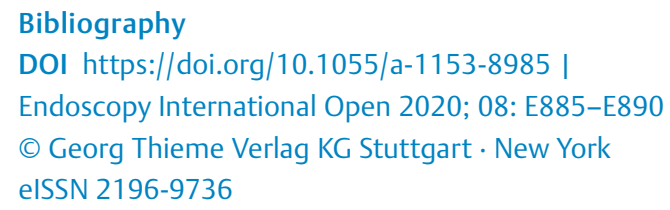

Corresponding author

Maximilien Barret, Department of Gastroenterology, Cochin Hospital, Paris, 27, rue du Faubourg St Jacques, 75014 Paris, France

Fax: +33-1-58411965

maximilien.barret@aphp.fr

\section{ABSTRACT}

Background and study aims Endoscopic treatment of Zenker's Diverticulum (ZD) using a flexible endoscope and a diverticuloscope consists of myotomy of the cricopharyngeus muscle, sparing the lower part of the diverticular septum. However, recurrence occurs in up to $54 \%$ of patients at
4 years. We assessed the feasibility and safety of a complete septotomy in endoscopic treatment of ZD.

Patients and methods We conducted a retrospective analysis of a prospectively collected database at a single referral center. All consecutive patients treated by complete resection of the diverticular wall were included. The endoscopic technique used a distal attachment cap and division of the ZD septum using a Dual Knife or a Triangle Tip knife in endocut mode, until the esophageal muscularis propria was seen and no residual diverticulum remained. Symptoms were evaluated using the Augsburger questionnaire.

Results Nineteen patients, 10 of whom were men with mean age $79 \pm 12$ years, were treated by complete septotomy for a symptomatic ZD with a median size of $2.5 \mathrm{~cm}$ (range $1-5 \mathrm{~cm}$ ). The clinical success rate was $100 \%$ and the complication rate was $10 \%$ (one pneumonia and one atrial fibrillation). Median hospital stay was 2 days (range 1-3 days). On Day 1 esophagogram, no extraesophageal contrast leakage was seen, periesophageal $\mathrm{CO}_{2}$ was still visible in two patients, and complete ZD regression was seen in $63 \%$ of patients. The 6 -month clinical success rate was $100 \%$, with two patients lost to follow-up, and a median symptom score of 0 (range $0-4$ ). After a mean \pm SD follow-up of $9 \pm 5$ months, the clinical success rate was $94 \%$ (16/17).

Conclusion Complete endoscopic septotomy is a feasible and safe therapeutic modality in patients with symptomatic ZD that does not require use of a diverticuloscope, and with good short-term efficacy. The complete regression of the diverticulum observed on Day 1 in $63 \%$ of patients could be a marker of long-term clinical success.

\section{Introduction}

Zenker's diverticulum (ZD) concerns the esophagus cervical region and corresponds to herniation of the mucosa and submucosa through the Killian Triangle, a zone of weakness in the posterior wall of the pharynx that is limited inferiorly by the cricopharyngeus muscle and laterally by the thyropharyngeus (or inferior pharyngeal constrictor) muscle [1]. Incidence of ZD is 2 per 100,000 people per year [2] The main symptoms include dysphagia, odynophagia, and regurgitation of undigested food, and may include sensations of choking, chronic coughing, and episodes of aspiration (with associated risks of pulmonary infection).

Initial treatment of ZD involves open surgery, with a combination of cricopharyngeal myotomy and suspension or resection of the diverticular pouch. However, the complication rates of this treatment approach, including postoperative mortality, have led to a preference for endoscopic treatment [3]. A recent 

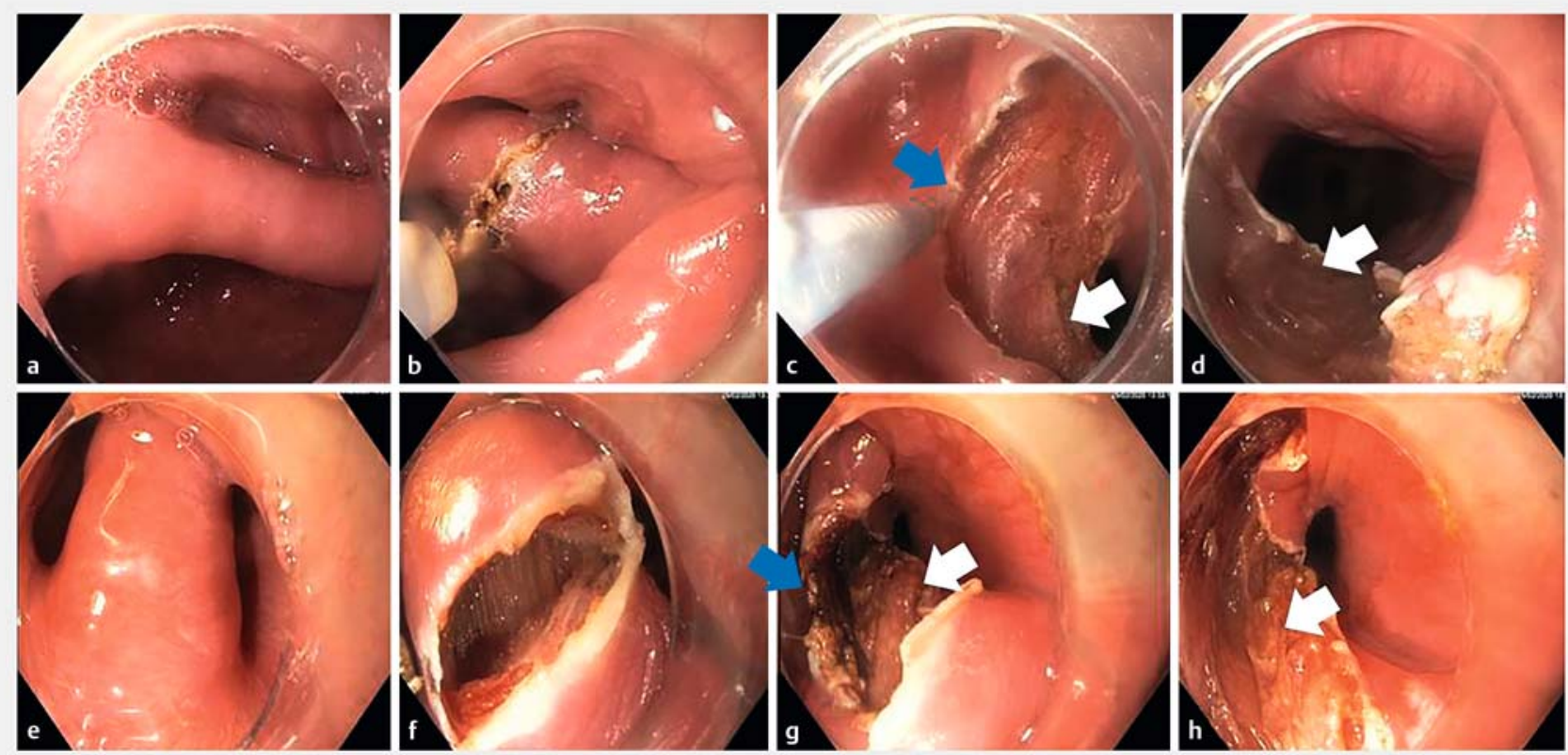

- Fig. 1 a,e Exposure of the diverticular septum. Diverticulum is in the 6 o'clock and e 9 o'clock positions. a Note the stenosed esophageal lumen in the 12 o'clock position and $\mathbf{e}$ the 3 o'clock position. $\mathbf{b}, \mathbf{f}$ Incision of the mucosa of the diverticular septum. $\mathbf{f}$ Visualization of the mucosa, submucosa, and cricopharyngeus muscle fibers. $\mathbf{c , d}, \mathbf{g}, \mathbf{h}$ Complete section of the diverticular septum beyond the cricopharyngeus muscle fibers before the bottom of the diverticulum is reached (blue arrows) and incision of the esophageal muscularis propria (white arrows). d Note the complete regression of the diverticulum and wide esophageal lumen opening.

meta-analysis demonstrated that endoscopic treatment of ZD, when compared with open surgical treatment, results in shorter procedure times and hospital stays and fewer complications. However, we observed approximately three times more recurrence after endoscopic treatment vs. surgery [4]. In the early 2000 s, flexible endoscopic treatment of ZD using a soft overtube called a diverticuloscope and performed by gastroenterologists was introduced $[5,6]$, and it has gained momentum ever since [7]. This approach, described as a myotomy of the cricopharyngeus muscle, is safer for the lower portion of the diverticular septum, because it is less likely to cause perforation and the positioning of the diverticuloscope prevents complete cutting of the diverticular septum. Although it is safe and easy to perform, this treatment has been associated with high rates of recurrences, reaching $54 \%$ at 4 years [8]. Factors associated with ZD recurrence are large size $(>50 \mathrm{~mm})$ of the diverticulum and a short $(<25 \mathrm{~mm})$ myotomy, and the existence of a residual diverticula after the myotomy [8]. Recently, improved mastery of endoscopic submucosal dissection techniques, costs of the diverticuloscope, and technical concerns about placement of the diverticuloscope in certain small diverticula or in patients with kyphosis or cervical osteoarthritis have led certain teams to abandon the diverticuloscope [9]. As an alternative, there is now consideration for complete septotomy of the diverticular wall. We sought to evaluate the feasibility and safety of endoscopic treatment with complete septotomy of ZD.

\section{Patients and methods}

This was a retrospective analysis of a prospectively collected database at a referral center for therapeutic digestive endoscopy. We included consecutive patients treated with a flexible endoscope, no diverticuloscope, and complete septotomy of the diverticular wall. Demographic data, medical history and specifically prior treatments for ZD, anticoagulant or antiplatelet medication, clinical symptoms, and general health status were recorded. Symptoms were graded using the Augsburg symptom score dedicated to the ZD [10] and comorbid conditions with the Charlson Index [11]. Malnutrition was defined by a body mass index $<18.5 \mathrm{Kg} / \mathrm{m}^{2}$ [12]. Complications and their severity were recorded and graded as recommended by the American Society for Gastrointestinal Endoscopy (ASGE) [13].

The procedure was performed by gastroenterologists $(n=3)$ with expertise in therapeutic endoscopy who had performed more than 20 ZD diverticulotomies. It was done with the patient under general anesthesia with orotracheal intubation and in left lateral or supine position. A standard gastroscope (GIFHQ 190, Olympus, Tokyo, Japan or EG-600 WR, Fujifilm, Tokyo, Japan) equipped with a distal attachment cap (D-201, Olympus, Tokyo, Japan) and an endoscopic submucosal dissection knife (TT knife or Dual Knife J, Olympus, Tokyo, Japan) was used. The procedure was carried out under $\mathrm{CO} 2$ insufflation and an antibiotic prophylaxis was administered with $2 \mathrm{~g} \mathrm{IV}$ amoxicillin-clavulanate.

Three experienced endoscopists completed endoscopic incision of the diverticular septum, through the mucosa, submuco- 


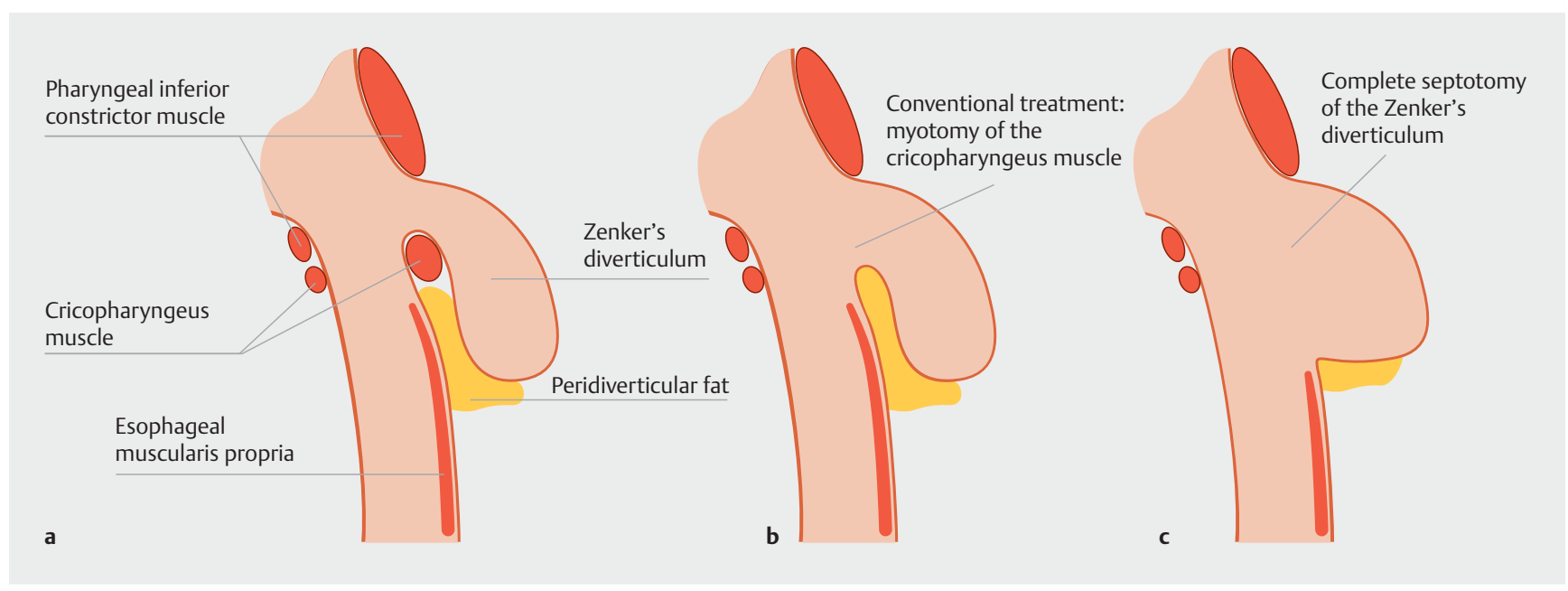

- Fig. 2 a lllustration of anatomical landmarks of Zenker's diverticulum, b conventional myotomy of the cricopharyngeus muscle, $\mathbf{c}$ and complete septotomy.

sa cricopharyngeus muscle, and connective tissue. The technique, illustrated in $\boldsymbol{\nabla}$ Fig. $\mathbf{1}$ and $\boldsymbol{\nabla}$ Fig. $\mathbf{2}$, consisted of exposure of the diverticular septum with the cap, sectioning the diverticular septum in endocut or spray coagulation mode until the diverticulum was completely obliterated, and visualizing the esophageal muscularis propria at the anal side of the incision and the bottom of the diverticulum at the oral side of the incision. A complete septotomy was obtained in the absence of residual diverticulum septum, allowing complete marsupialization of the diverticulum and easy passage of the endoscope in the esophagus ( $\vee$ Video 1 ). Then, from none to four hemoclips were placed, according to the operator's judgment, to join the mucosa of the bottom of the diverticulum with the muscularis propria of the esophagus. After the procedure, patients received systematic antibiotic treatment with amoxicillin-clavulanate for 3 days. All patients were kept nil per os until a systematic follow-up radiological imaging with oral contrast intake on the first post-procedure day to rule out an esophageal fistula and assess for presence of a residual diverticulum. In the absence of contrast leakage, patients resumed oral soft food and were discharged the second postoperative day. A follow-up visit with a new esophagogram was scheduled at 3 months. Afterwards, clinical results were collected by telephone.

\section{Results}

From February 2018 to August 2019, 19 patients (10 men, 9 women, mean \pm SD age $79 \pm 12$ years) underwent flexible endoscopic treatment for ZD with complete septotomy in our hospital. No technical failure was encountered. Cardiovascular comorbid conditions, such as coronary artery disease, congestive heart failure, and hypertension, were reported in 16 patients, and pulmonary insufficiency in four. Detailed patient characteristics are presented in $>$ Table $\mathbf{1}$.

Seven patients had a history of cervical surgery such as thyroidectomy $(n=5)$, excision of a parathyroid adenoma $(n=1)$ or cervicotomy for ZD $(n=1)$. Six patients had previously been

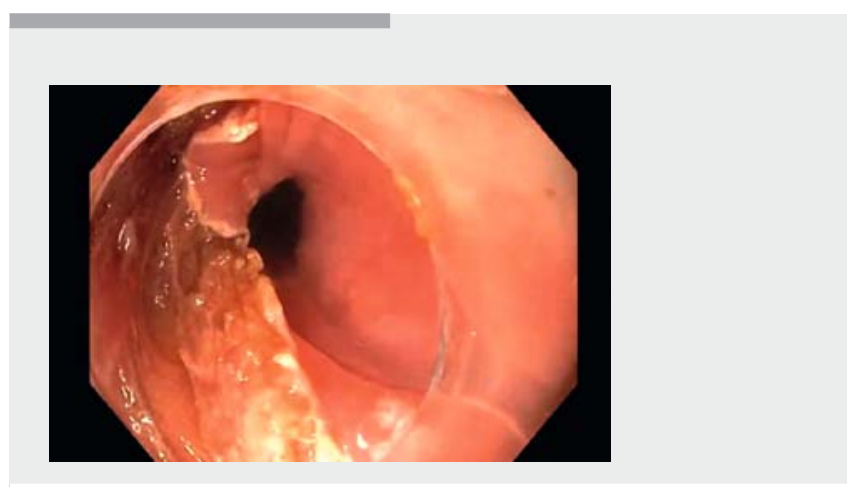

Video 1 Endoscopic view after complete endoscopic septotomy for Zenker's diverticulum. Note the easy passage in the esophageal lumen, absence of residual diverticulum with incision of the bottom of the diverticular mucosa, opening of the esophageal muscularis propria, and peridiverticular fat in between.

treated for ZD with surgery or flexible endoscopy. Sixteen patients reported moderate to severe dysphagia and 14 pharyngo-oral regurgitation with a median (range) Augsburg's score of 6.5 (range 4-10). The mean \pm SD duration of symptom evolution was $2.5 \pm 1.6$ years. Six patients $(31 \%)$ had complications from the $Z D$, such as malnutrition $(n=4)$ or aspiration pneumonia $(n=2)$.

The median (range) size of diverticulum was $2.5 \mathrm{~cm}$ (range $1-5)$. The technical success rate was $100 \%$. However, complications occurred in $10 \%$ of patients (one aspiration pneumonia and one atrial fibrillation complicating a dehydration requiring one-night intensive care admission), one of which (5\%) was severe according to the ASGE grading system. The median (range) hospital stay was 2 days (1-3). Procedural characteristics are detailed in > Table 2 .

All 19 patients had radiological imaging with water-soluble oral contrast on day 1 . For logistic reasons, an esophagogram was performed in 11 patients and a computed tomography scan in the remaining eight. The diverticulum was completely 
- Table 1 Patient characteristics $(n=19)$.

\begin{tabular}{|c|c|}
\hline Age-mean $\pm S D$ & $79 \pm 12$ \\
\hline Male/female-n & $10 / 9$ \\
\hline \multicolumn{2}{|l|}{ Major comorbid conditions - n (\%) } \\
\hline - Cardiovascular & $16(84)$ \\
\hline - Pulmonary & $4(21)$ \\
\hline - Other ${ }^{1}$ & $6(31)$ \\
\hline Charlson Cormorbidity Index - median (range) & $4(1-7)$ \\
\hline Anticoagulation - $\mathrm{n}(\%)$ & $4(21)$ \\
\hline Antiaggregation - (\%) & $6(31)$ \\
\hline Cervical History-n (\%) & $7(36)$ \\
\hline Cervical surgery ${ }^{2}$ & $7(36)$ \\
\hline Radiotherapy & $0(0)$ \\
\hline Other & $0(0)$ \\
\hline ZD treatment history $-\mathrm{n}(\%)$ & $6(31)$ \\
\hline Flexible endoscope and diverticuloscope & $4(21)$ \\
\hline Flexible endoscope without diverticuloscope & $2(10)$ \\
\hline Surgery & $1(5)$ \\
\hline $\begin{array}{l}\text { Duration of evolution of symptoms in year- } \\
\text { mean } \pm \text { SD }\end{array}$ & $2.5 \pm 1.6$ \\
\hline \multicolumn{2}{|l|}{ Symptoms - n (\%) } \\
\hline - Dysphagia & $16(84)$ \\
\hline - Regurgitation & $14(73)$ \\
\hline Augsburg's Score - median (range) & $6.5(4-10)$ \\
\hline Complications - n (\%) & $6(31)$ \\
\hline Malnutrition & $4(21)$ \\
\hline Aspiration pneumonia & $2(10)$ \\
\hline
\end{tabular}

SD, standard deviation; ZD, Zenker's diverticulum

${ }^{1}$ Hypothyroidism $(n=3)$, Horton's disease $(n=1)$, esophageal stenosis $(n=1)$ and uterine cancer $(n=1)$

2 Thyroidectomy $(n=5)$, excision of a parathyroid adenoma $(n=1)$ and cervicotomy for Zenker's diverticulum $(n=1)$
- Table 2 Procedural characteristics.

Diverticulum size in $\mathrm{cm}$ - median (range)

\begin{tabular}{|l|l|}
\hline - Endoscopy & $2.5(1-4)$ \\
\hline - Radiology & $2.5(1-5)$ \\
\hline
\end{tabular}

Technical success $-\mathrm{n}(\%)$

$19(100)$

ESD knife $-\mathrm{n}(\%)$

- Dual knife 2(10)

- TT knife $17(89)$

Number of clips - median ( range) $2(0-4)$

Complications $-\mathrm{n}(\%)^{1} \quad 2(10)$

Hospital stay in days - median (range)

$2(1-4)$

ESD, endoscopic submucosal dissection; TT, triangle tip

11 aspiration pneumonia and 1 transition to atrial fibrillation complicating dehydration

cleared in 12 patients (63\%). On the eight scans performed, there was evidence of asymptomatic periesophageal leakage of $\mathrm{CO} 2$ in two cases, without any oral contrast leakage or clinical consequence.

The clinical success rate at 6 months was $100 \%$, with a median symptom score of 0 (range $0-4$ ). However, two patients were lost to follow-up, one patient reported a partial clinical and radiological recurrence at 6 months, and another at 10 months. Six of the 7 patients (85\%) with a residual diverticulum on Day 1 esophagogram had complete clinical remission at 6 months. After a medium follow-up of $9 \pm 5$ months, the clinical success rate was $84 \%$ in intention to treat and $94 \%$ in per protocol. No patient required further endoscopic or surgical treatment to date. Patient outcomes are detailed in $>$ Table 3.

\section{Discussion}

In this study, we showed the feasibility, safety and efficacy of a complete septotomy for flexible endoscopic treatment of ZD. We achieved $100 \%$ short-term symptom relief, and $94 \%$ symptoms relief at the latest follow-up visit, without any local com-

- Table 3 Patient outcomes.

\begin{tabular}{|l|c|c|}
\hline & Intention to treat $(\mathbf{n = 1 9 )}$ & Per protocol (n=17) \\
\hline Residual diverticulum $-\mathrm{n}(\%)$ & $7(37)$ & $7(47)$ \\
\hline Clinical success at 6 months $-\mathrm{n}(\%)$ & $17(89)$ & $17(100)$ \\
\hline - Partial & $9(47)$ & $9(53)$ \\
\hline - Complete & $8(42)$ & $8(47)$ \\
\hline Augsburg's score at 6 months - median (range) & $0(0-4)$ & $0(0-4)$ \\
\hline Recurrence of the diverticulum at 6 months - $\mathrm{n}(\%)$ & $1(5)$ & $1(6)$ \\
\hline Clinical success at last follow-up - $\mathrm{n}(\%)$ & $16(84)$ & $16(94)$ \\
\hline
\end{tabular}


plications. These numbers are comparable to other studies of flexible endoscopic treatment of ZD [7]. However, given the elderly and frail population included in our study, with $20 \%$ malnourished patients and $84 \%$ with severe cardiovascular comorbid conditions, we observed $10 \%$ medical complications of the anesthesia, of which $5 \%$ were severe.

In a retrospective analysis of 76 patients treated surgically or endoscopically for ZD, Shah et al. showed endoscopic staplerassisted diverticulotomy to be associated with a longer residual diverticular wall and with higher risk of recurrent disease [14]. Similarly, Costamagna et al., in a study of 89 patients, correlated risk of recurrent disease with a $<25 \mathrm{~mm}$ length of septotomy [8]. However, even this extremely experienced team has not changed their ZD endoscopic treatment technique, and they continue to perform septotomy limited to the mucosa and muscle fibers, without taking into account residual diverticular septum [8], probably to avoid perforation of the posterior wall of the pharynx.

The question of recurrence has been tackled with several approaches. Some teams chose to resect instead of cutting the cricopharyngeus muscle $[10,15]$ to prevent muscle reparation, but we chose to increase the length of the septal incision. This approach also leads to a wide opening of the cricopharyngeus, and to exposure of fatty and connective tissue to saliva and food, because the size of the mucosal defect often does not allow for clip closure. Of note, while endoscopic ZD treatments were initially performed with needle knives, we used endoscopic submucosal dissection knives, as they have been shown to be safe, precise and effective for this indication [16]. Using the peroral endoscopic myotomy technique, Li et al recently performed a complete septotomy through a submucosal tunnel, with the idea of protecting the site of the septotomy with healthy mucosa $[17,18]$. Our data suggest that this cumbersome procedure is probably unnecessary, since no local infection of oral contrast leakage is evidenced after complete septotomy.

Unexpectedly, despite endoscopic complete obliteration of the diverticulum immediately after the septotomy and under insufflation, we observed a residual diverticulum on Day 1 esophagogram in almost one of three patients. This could be explained by the persistence of the posterior diverticular wall, rather than by a residual septum. Presence of a residual diverticulum on Day 1 esophagogram does not seem to be predictive of early symptom recurrence. Indeed, we observed a complete clinical success at 6 months in almost $85 \%$ of patients with a residual diverticulum on Day 1 esophagogram. However, despite septotomy, two patients developed actual endoscopic, radiologic and clinical recurrence of ZD, one at 3 months, and the other at 10 months. This suggests that an improved understanding of the pathophysiology of ZD and the molecular changes occurring in the cricopharyngeus muscle is needed to diminish the recurrence rates.

The main limitations of our study are the small number of patients included and short-term follow-up data that do not allow us to draw conclusions on the long-term efficacy of complete septotomy. Furthermore, in less than a year, we have already lost two patients to follow-up. Their age or the distance between their homes and the endoscopy center where they are treated may explain the absence of follow-up visits or even follow-up telephone calls in some cases. Of note, almost one-third of the patients in our study had a previously treated ZD. ZD recurs in almost half of patients treated endoscopically, so these numbers reflect daily practice in management of this patient population. Although this brings heterogeneity to the study population, it could be argued that the possibility of achieving technical and clinical success in patients with treatment failure or recurrences is mandatory for any innovative ZD treatment.

\section{Conclusion}

Complete endoscopic septotomy of ZD is technically feasible without increased morbidity, as compared to conventional cricopharyngeal myotomy. Complete clearing of the diverticulum observed in $63 \%$ of cases could be associated with improved long-term symptom relief.

\section{Competing interests}

The authors declare that they have no conflict of interest.

\section{References}

[1] Van Overbeek J]. Meditation on the pathogenesis of hypopharyngeal (Zenker's) diverticulum and a report of endoscopic treatment in 545 patients. Ann Otol Rhinol Laryngol 1994; 103: 178-185

[2] Law R, Katzka DA, Baron TH. Zenker's Diverticulum. Clin Gastroenterol Hepatol 2014; 12: 1773-1882; quiz e111-e112

[3] Verdonck J, Morton RP. Systematic review on treatment of Zenker's diverticulum. Eur Arch Otorhinolaryngol 2015; 272: 3095-3107

[4] Albers DV, Kondo A, Bernardo WM et al. Endoscopic versus surgical approach in the treatment of Zenker's diverticulum: systematic review and meta-analysis. Endosc Int Open 2016; 4: E678-E686

[5] Mulder C], Costamagna G, Sakai P. Zenker's diverticulum: treatment using a flexible endoscope. Endoscopy 2001; 33: 991-997

[6] Evrard S, Le Moine O, Hassid S et al. Zenker's diverticulum: a new endoscopic treatment with a soft diverticuloscope. Gastrointest Endosc 2003; 58: 116-120

[7] Ishaq S, Hassan C, Antonello A et al. Flexible endoscopic treatment for Zenker's diverticulum: a systematic review and meta-analysis. Gastrointest Endosc 2016; 83: 1076-1089.e5

[8] Costamagna G, lacopini F, Bizzotto A et al. Prognostic variables for the clinical success of flexible endoscopic septotomy of Zenker's diverticulum. Gastrointest Endosc 2016; 83: 765-773

[9] Bresteau C, Barret M, Guillaumot M-A et al. Do we still need a diverticuloscope for the flexible endoscopic septotomy of Zenker's diverticulum? J Gastroenterol Hepatol 2020; 35: 630-633. doi:10.1111/ jgh.14923

[10] Goelder SK, Brueckner J, Messmann H. Endoscopic treatment of Zenker's diverticulum with the stag beetle knife (sb knife) - feasibility and follow-up. Scand J Gastroenterol 2016; 51: 1155-1158

[11] Charlson ME, Pompei P, Ales KL et al. A new method of classifying prognostic comorbidity in longitudinal studies: development and validation. J Chronic Dis 1987; 40: 373-383 
[12] Cederholm T, Bosaeus I, Barazzoni R et al. Diagnostic criteria for malnutrition - An ESPEN Consensus Statement. Clin Nutr 2015; 34: 335340

[13] Cotton PB, Eisen GM, Aabakken L et al. A lexicon for endoscopic adverse events: report of an ASGE workshop. Gastrointest Endosc 2010; 71: $446-454$

[14] Shah RN, Slaughter KA, Fedore LW et al. Does residual wall size or technique matter in the treatment of Zenker's diverticulum? Laryngoscope 2016; 126: 2475-5479

[15] Ishaq S, Battaglia G, Antonello A. Double incision and snare resection in symptomatic Zenker's diverticulum: a modification of the stagbeetle knife technique. Endoscopy 2018; 50: 182
[16] Laquière A, Grandval P, Arpurt JP et al. Interest of submucosal dissection knife for endoscopic treatment of Zenker's diverticulum. Surg Endosc 2015; 29: 2802-2810

[17] Li Q-L, Chen W-F, Zhang X-C et al. Submucosal Tunneling endoscopic septum division: a novel technique for treating Zenker's diverticulum. Gastroenterology 2016; 151: 1071-1074

[18] Yang J, Novak S, Ujiki M et al. An international study on the use of peroral endoscopic myotomy in the management of Zenker's diverticulum. Gastrointest Endosc 2020; 91: 163-168 